\title{
The modified Bers conjecture
}

\author{
L. Escauriaza \\ Universidad del Pais Vasco \\ mtpeszul@lg.ehu.es
}

It is well known that on any 2-dimensional Lipschitz domain $D$, there are no non-constant harmonic functions whose gradient vanishes on a set with positive arc-length measure contained in the boundary of $D$. On the contrary, when the dimension is greater than 2, these bad harmonic functions do exist. In fact, in [3] the authors constructed a non-zero harmonic function $u$ defined in the upper closed space $\mathbb{R}_{+}^{3}$, verifying that both $u$ and its gradient vanish simultaneously on a set with positive 2-dimensional Lebesgue measure contained in the boundary of $\mathbb{R}_{+}^{3}$. This counterexample disproved the so called "Bers' conjecture", which claimed that these bad harmonic functions could not exist.

On the other hand, the following "modified Bers conjecture" still remains an open question:

Let $u$ be a harmonic function in a connected Lipschitz domain $D$ in $\mathbb{R}^{d}$, $d \geq 3$, containing the origin as a boundary point. Assume that $u$ vanishes continuously on $B_{2 R} \cap \partial D$ for some positive $R$. Then, $u$ must be identically zero in $D$ if the surface measure of the set

$$
E=\left\{Q \in \partial D \cap B_{R}: \nabla u(Q)=0\right\}
$$

is positive.

Observe that in this case, one assumes that the tangential derivatives of $u$ are identically zero on an open set of the boundary, and one is asking what can happen with the size of the set where the normal derivative vanishes within the open set.

This conjecture is known to hold when the boundary of $D$ can be written locally as the graph of functions $\varphi: \mathbb{R}^{d-1} \longrightarrow \mathbb{R}$ verifying that for some $M<\infty, 0<\alpha \leq 1$ and all $x, y$ in $\mathbb{R}^{d-1}$

$$
(x-y) \cdot \nabla \varphi(x)-(\varphi(x)-\varphi(y)) \geq-M|x-y|^{1+\alpha} .
$$

In particular, this property holds when the functions $\varphi$ are the sum of a $C^{1, \alpha}$ function and a convex function [1],[2], [8]. Moreover, when $D$ is a $C^{1, \alpha}$ domain and $u$ is a nonzero harmonic function verifying the above conditions, it has been 
shown that the Hausdorff dimension of the set $E$ is not greater than $d-2$ [9], [2]. But nothing is known about this problem when $D$ is just a $C^{1}$ domain.

This is an interesting and challenging problem in harmonic analysis, and a positive answer to the modified conjecture would have some interesting applications to control theory [7]. For instance, it would be possible to yield exact controllability for the heat operator on cylindrical domains with minimal smoothness conditions on the base of the cylinder. As shown by Lin in [9], uniqueness for boundary problems can also be used to obtain estimates for nodal sets of eigenfunctions due to Donnelly and Fefferman [4].

The theorems that have been proved so far rely either on a monotonicity method introduced by Garofalo and Lin [5] or Carleman inequalities. An exposition of the Carleman method and related conjectures can be found in [6].

\section{References}

[1] Adolfsson A., Escauriaza E., Kenig C.E., Convex domains and unique continuation at the boundary, Rev. Mat. Iberoamericana 11,3 (1995),513-525.

[2] Adolfsson A., Escauriaza E., $C^{1, \alpha}$ domains and unique continuation at the boundary, Comm. Pure. Appl. Math. Vol. L (1997),935-969.

[3] Bourgain J., Wolff T., A remark on gradients of harmonic functions in dimension $d \geq 3$, Colloquium Mathematicum LX/LXI (1990), 253-260.

[4] Donnelly H. and Fefferman C., Nodal sets of eigenfunctions on Riemannian manifolds, Inv. Math. 93 (1988), 161-183.

[5] Garofalo N., Lin F.H., Monotonicity properties of variational integrals, $A_{p}$-weights and unique continuation, Indiana Univ. Math J. 35 (1986), 245-267.

[6] Jerison D. and Lebeau G. Nodal sets of sums of eigenfunctions, to appear in "Harmonic Analysis and Partial Differential Equations: Essays in Honor of Alberto Calderón," ed. Michael Christ, Carlos Kenig, and Cora Sadosky, Chicago U. Press, 1999.

[7] Kenig C.E., Spring School 94: Potential theory and analysis , Paskey nad Jizerou (Czech Republic). Lectures by Carlos E. Kenig. Notes by Michael Korey.

[8] Kukavica I. and Nyström K., Unique continuation on the boundary for Dini domains, to appear Proc. A. M. S.

[9] Lin F.-H., Nodal sets of solutions of elliptic and parabolic equations , Comm. Pure and Appl. Math. XLIV (1991), 287-308. 